\title{
Extracting (pion) form factors from finite volume lattices
}

\author{
Hidenori Fukaya* and Takashi Suzuki for JLQCD collaboration \\ Department of Physics, Osaka University, Toyonaka, Osaka 560-0043 Japan \\ E-mail: hfukava@het.phys.sci.osaka-u.ac.ip
}

In lattice QCD simulations, there is a strong tendency to over-estimate the form factors (and therefore, under-estimate the related charge radii) of pions and other hadrons. The three major systematic in lattice QCD: finite lattice spacing, heavier pion mass, and finite volume all could contribute to the over-estimation of the form factors. Using the chiral effective theory, this can be easily understood as a distortion of the chiral logarithm. If a chiral symmetric Dirac operator is employed for lattice QCD, one has a good control of the former two, while the volume has to be small due to its high numerical cost. In this talk, we focus on the finite volume effects using chiral perturbation theory and discuss how to avoid the over-estimation of the (pion) form factors. We also present our recent lattice results with dynamical chiral fermions.

The 8th International Workshop on Chiral Dynamics, CD2015 ***

29 June 2015 - 03 July 2015

Pisa,Italy

\footnotetext{
* Speaker.
} 


\section{Introduction}

Recent developments of computational resources and numerical algorithms allow us to simulate lattice QCD in realistic setups (we refer the readers to a review article [四). In particular, it is remarkable that some of the simulations have already reached at the physical pion mass. In these simulations, the hadronic two-point functions in many different channels are computed and it has been confirmed that the low-energy hadron spectrum is (almost) consistent with the currently known experimental values.

Our next non-trivial target is the three-point functions. In this talk, we focus mainly on that of the pion which is relevant for the computation of its electro-magnetic form factors. Experimentally, it is related to the pion charge radius $\left\langle r^{2}\right\rangle_{V}$ through the relation

$$
\left\langle r^{2}\right\rangle_{V}=\left.6 \frac{d F_{V}\left(q^{2}\right)}{d q^{2}}\right|_{q^{2}=0},
$$

where $F_{V}\left(q^{2}\right)$ denotes the electro-magnetic form factor at the momentum transfer $q^{2}$ (in Minkowski space-time). In terms of chiral perturbation theory (ChPT), it is related to the low-energy constant $L_{9}$ (or $l_{6}$ in the $S U$ (2) case), which appears at the next-to-leading order (NLO) in the chiral Lagrangian [Z, B]].

The electro-magnetic pion form factor and its charge radius have been computed by many lattice QCD simulations (we refer the readers to a review in [田]). In recent developments (after the last workshop Chiral Dynamics 2012), it is notable that the simulated pion mass has been reached near the physical point $[[5, \mathbb{Q}, \square,[8,0]$. Moreover, the use of the twisted boundary condition on the valence quark becomes standard, which allows us to study the pion form factors with arbitrarily small momentum transfers. We also note that the possible systematic errors due to the twisting and partially quenching effect due to having the periodic boundary in the sea quark sector, have been investigated by Ref. [[0]]. Even with these developments, however, the lattice data of the pion charge radius are still sizably lower than the experimental value $\left\langle r^{2}\right\rangle_{V}=0.452(11) \mathrm{fm}^{2}$ [ए]].

Not only in the pion form factors but also in those of other hadrons, there is a strong tendency of lattice QCD simulations to under-estimate the various charge radius. In the computation of the

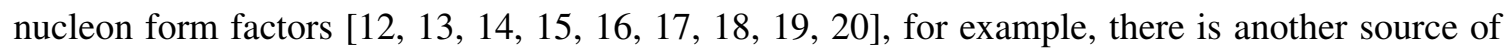
the systematic errors which could lead the under-estimation. It is the contamination by the excited states. At long range, the nucleon propagator is known to be very noisy, which makes it difficult to obtain a clean signal removing the excited state's effect.

In this talk, we would like to understand using the chiral effective theory, why the hadron charge radii are generally likely to be under-estimated, or equivalently, why the corresponding form factors are over-estimated. As will be shown later, the major three systematics in lattice QCD, finite lattice spacing, lack of chiral symmetry, and finiteness of the volume size all could lead to the under-estimation.

Then we report on the recent progress in our JLQCD collaboration on the pion form factors. Our strategy is to use the dynamical chiral fermions, which allows us to reliably use chiral perturbation theory (ChPT) in the same form as in the continuum theory, and at the same time, automatically remove possible $\mathscr{O}(a)$ ( $a$ denotes the lattice spacing) errors [प, [प]]. 
Instead, the volume size of our simulations is quite limited due to the high numerical cost. Namely, the finite volume effect is our main concern in extracting the pion form factors. Here, we propose a method to automatically cancel the main contribution from the zero momentum mode of pions and demonstrate that the pion charge radius can be extracted reasonably from our small lattice.

\section{Why charge radii under-estimated ?}

It is not very difficult to understand why many of the hadron charge radii are likely to be under-estimated. Since the pion becomes exactly massless in the chiral limit, there should be a logarithmic enhancement, or the so-called chiral logarithm in any form factors unless a non-trivial cancellation occurs. Therefore, any introduction of extra mass scale $\delta m^{2}$ could be the source for the under-estimation of the charge radii, since it would play the role of an infra-red cut-off,

$$
\ln \left[M_{\pi}^{2}\right] \rightarrow \ln \left[M_{\pi}^{2}+\delta m^{2}\right] .
$$

In particular, the three major lattice systematics, the lattice discretization effect $\delta m \sim \Lambda_{\mathrm{QCD}}^{2} a$, the heavier simulated pion mass $\delta m \sim M_{\pi}^{\text {simulated }}-M_{\pi}$, and the finite volume effect $\delta m \sim 1 / V^{1 / 4}$, all could contribute to this under-estimation.

A beautiful example was given in ChPT [22] where the finite volume correction to the pion one-loop were computed,

$$
\frac{M_{\pi}^{2}}{16 \pi^{2}} \ln M_{\pi}^{2}+g_{1}\left(M_{\pi}^{2}\right)
$$

where $g_{1}\left(M_{\pi}^{2}\right)$ denotes the finite volume correction, which is exponentially small when the mass $M_{\pi}$ is large compared to the size of the volume (in the $p$ regime),

$$
g_{1}\left(M_{\pi}^{2}\right)=\sum_{a=\left(n_{1} L, n_{2} L, n_{3} L, n_{4} T\right) \neq 0}^{\infty} \frac{\sqrt{M_{\pi}^{2}}}{4 \pi^{2}|a|} K_{1}\left(\sqrt{M_{\pi}^{2}}|a|\right)
$$

but near the chiral limit (in the $\varepsilon$ regime) it becomes

$$
g_{1}\left(M_{\pi}^{2}\right)=-\frac{M_{\pi}^{2}}{16 \pi^{2}} \ln \left(M_{\pi}^{2} V^{1 / 2}\right)-\sum_{n=1}^{\infty} \frac{\beta_{n}}{(n-1) !} M_{\pi}^{2(n-1)} V^{(n-2) / 2}+\frac{1}{M_{\pi}^{2} V} .
$$

Here $K_{1}$ is the modified Bessel function and the summation is taken over the 4-vector $a_{\mu}=n_{\mu} L_{\mu}$ with $L_{i}=L(i=1,2,3)$ and $L_{4}=T . \beta_{i}$ 's denote the shape coefficients defined in [23]]. It is the first term of Eq. (2.4) that exactly cancels the original logarithmic divergence of Eq. (L2.2). The last term should be replaced by the exact zero-mode integrals in the $\varepsilon$ expansion.

\section{How to remove finite volume effect}

In quantum field theories, finite volume effects are produced by the propagation of particles, wrapping around the finite volume. It is not difficult to understand that the lighter particles give the heavier contribution. In QCD, it is the pion that gives the dominant contribution. For a lattice size $L=2 \mathrm{fm}$, for instance, the pion could give a sizable propagation $\exp \left(-M_{\pi} L\right) \sim 0.25$, while that of kaon (with its mass $M_{K}$ ) gives only $\exp \left(-M_{K} L\right)<0.01$. 
For this reason, we use the value of $M_{\pi} L$ as the indicator of how much the finite size effects are under control in a lattice QCD simulation. The criterion adopted by FLAG [24] is

good : $M_{\pi, \min } L>4$ or at least 3 volumes,

soso : $M_{\pi, \min } L>3$ or at least 2 volumes,

bad : otherwise.

To obtain the "good" score, one needs to suppress the pion propagation to $\exp \left(-M_{\pi} L\right) \sim 0.018$ and needs $L=5.8 \mathrm{fm}$ when we want to simulate QCD at the physical pion mass, which is still a challenging size for up-to-date supercomputers.

In this talk, we would like to look further in details of the dominance of the pion in the finite volume effects. In fact, the non-zero momentum modes of the pions can not be crucial to the effects. Since the momenta are discrete in a finite volume, non-zero momentum modes of the pion only give

$$
\exp \left(-\sqrt{M_{\pi}^{2}+\mathbf{p}^{2}} L\right) \leq \exp \left(-\sqrt{M_{\pi}^{2}+(2 \pi / L)^{2}} L\right) \leq \exp (-2 \pi)=0.0019 .
$$

Namely, among the different modes of the pions, only the zero-mode gives the dominant contribution to the finite volume effects.

Then an interesting possibility arises: if we could remove or reduce the effect of the zeromode, we could have a better control of the finite volume effects even on small "soso" or "bad" lattices. In the following, we will show this is actually the case.

First, we would like to note that the zero momentum mode of the pion is space-time independent. Its effect always comes as a $x$-independent constant or an overall constant of the $x$-dependent function. Namely, it always appears in a form

$$
f(x)=A+B g(x),
$$

where only $A$ and $B$ contain the corresponding finite volume effects from the zero-mode.

It is not difficult at all to remove these finite volume effect by simple arithmetics, subtraction and division :

$$
\frac{f(x)-f\left(x_{0}\right)}{f\left(x_{1}\right)-f\left(x_{0}\right)}=\frac{g(x)-g\left(x_{0}\right)}{g\left(x_{1}\right)-g\left(x_{0}\right)},
$$

where $x_{0}$ and $x_{1}$ are some appropriate reference points different from $x$. This simple idea applies to any complicated correlation functions in QCD. In the next section, we will compute more concrete examples of two and three-point functions within ChPT and explicitly show the cancellation of the zero-mode's finite volume effects.

\section{4. $\varepsilon$ expansion in ChPT}

The $\varepsilon$ expansion of ChPT [D2] is a best tool to understand how the zero-mode's cancellation can be achieved, since it separately and non-perturbatively treats the zero-momentum mode from the non-zero modes. The chiral field is expressed by

$$
U(x)=U_{0} \exp \left(i \frac{\sqrt{2} \pi(x)}{F}\right),
$$


where $U_{0} \in S U\left(N_{f}\right)$ denotes the zero-mode, while $\pi(x)$ denotes the non-zero mode under a constraint $\int d^{4} x \pi(x)=0$. Here, $F$ is the pion decay constant in the chiral limit.

The chiral Lagrangian is written as

$$
\mathscr{L}=-\frac{m \Sigma}{2} \operatorname{Tr}\left[U_{0}+U_{0}^{\dagger}\right]+\frac{1}{2} \operatorname{Tr}\left[\partial_{\mu} \pi(x)\right]^{2}+\frac{m \Sigma}{2 F^{2}} \operatorname{Tr}\left[\left(U_{0}+U_{0}^{\dagger}\right) \pi(x)^{2}\right],
$$

which describes a hybrid system of a matrix and massless bosonic fields. Here, $\Sigma$ denotes the chiral condensate.

Let us begin our computation with the pseudoscalar two-point function:

$$
\langle P(x) P(0)\rangle=A+B \sum_{p^{\prime} \neq 0} \frac{1}{V} \frac{e^{i p^{\prime} x}}{p^{\prime 2}}+C \sum_{p^{\prime} \neq 0} \frac{1}{V} \frac{e^{i p^{\prime} x}}{p^{\prime 4}},
$$

where $A, B$, and $C$ are the $x$-independent contribution from the zero mode. These are expressed by the modified Bessel functions of dimensionless quantity $m_{i} \Sigma V$ 's, and their explicit forms are known in the literature [25, [26, 27, [28]. Note here that the zero-mode is subtracted from the momentum summation to avoid overcounting and to keep the condition $\int d^{4} x \pi(x)=0$.

In order to eliminate $A$, we can make a "subtraction" $\Delta\langle P(x) P(0)\rangle \equiv\langle P(x) P(0)\rangle-\left\langle P\left(x_{\text {ref }}\right) P(0)\right\rangle$, where $x_{\text {ref }}$ denotes a reference point. We can also use the Fourier transform (as a more sophisticated "subtraction")

$$
C_{P P}^{2 \mathrm{pt}}(t, \mathbf{p}) \equiv \frac{1}{L^{3}} \int d^{3} x e^{-i \mathbf{p} \cdot \mathbf{x}}\langle P(x) P(0)\rangle,
$$

which automatically cancels the unwanted constant $A$ when the spatial momentum is non-zero, $\mathbf{p} \neq \mathbf{0}$. In the following, we will use $C_{P P}^{2 \mathrm{pt}}(t, \mathbf{p})$ for non-zero $\mathbf{p}$, and

$$
\Delta_{t} C_{P P}^{2 \mathrm{pt}}(t, \mathbf{p}=\mathbf{0}) \equiv C_{P P}^{2 \mathrm{pt}}(t, \mathbf{p}=\mathbf{0})-C_{P P}^{2 \mathrm{pt}}\left(t_{\mathrm{ref}}, \mathbf{p}=\mathbf{0}\right)
$$

for the zero-mode. For the reference point, we will use $t_{\text {ref }} \sim 1 \mathrm{fm}$.

Next, we take the ratios of the correlators with different momenta,

$$
\frac{C_{P P}^{2 \mathrm{pt}}(t, \mathbf{p})}{C_{P P}^{2 \mathrm{pt}}\left(t, \mathbf{p}^{\prime}\right)} \text { or } \frac{C_{P P}^{2 \mathrm{pt}}(t, \mathbf{p})}{\Delta_{t} C_{P P}^{2 \mathrm{pt}}\left(t, \mathbf{0}^{\prime}\right)},
$$

which cancel the second dominant finite volume effect from the zero-mode $B$. Of course, there remains $C$ and those in higher contributions but they should be suppressed typically by $\sim 1 /\left(4 \pi F^{2} \sqrt{V}\right)$.

Now let us compute our main target: the pseudoscalar-vector-pseudoscalar three-point function. At large distances in the temporal direction, the correlator with the initial non-zero momentum $\mathbf{p}_{i}$ and final non-zero momentum $\mathbf{p}_{f}$ can be written in a double cosh form

$$
\begin{aligned}
C_{P V_{0} P}^{3 \mathrm{pt}}\left(t, t^{\prime} ; \mathbf{p}_{i}, \mathbf{p}_{f}\right)= & B^{3 \mathrm{pt}}(m \Sigma V)\left[E\left(\mathbf{p}_{i}\right)+E\left(\mathbf{p}_{f}\right)\right] F_{V}\left(q^{2}\right) \\
& \times \cosh \left[E\left(\mathbf{p}_{i}\right)(t-T / 2)\right] \cosh \left[E\left(\mathbf{p}_{f}\right)\left(t^{\prime}-T / 2\right)\right],
\end{aligned}
$$

where the coefficient $B^{3 \mathrm{pt}}(m \Sigma V)$ contains the dominant finite volume effects. The vector form factor (which is equivalent to the electromagnetic form factor in our isospin symmetric case) is 
denoted by $F_{V}\left(q^{2}\right)$ with the four-momentum transfer $q^{2}=\left(\mathbf{p}_{f}-\mathbf{p}_{i}\right)^{2}-\left(E\left(\mathbf{p}_{f}\right)-E\left(\mathbf{p}_{i}\right)\right)^{2}$. By taking a ratio of the three-point and two two-point functions,

$$
R_{V}^{1}\left(t, t^{\prime} ;\left|\mathbf{p}_{i}\right|,\left|\mathbf{p}_{f}\right|, q^{2}\right) \equiv \frac{\frac{1}{N_{\left|\mathbf{p}_{i}\right|,\left|\mathbf{p}_{f}\right|}^{3 \mathrm{pt}}} \sum_{\text {fixed }\left|\mathbf{p}_{i}\right|,\left|\mathbf{p}_{f}\right|, q^{2}} \frac{C_{P V P}^{3 \mathrm{pt}}\left(t, t^{\prime} ; \mathbf{p}_{i}, \mathbf{p}_{f}\right)}{E\left(\mathbf{p}_{i}\right)+E\left(\mathbf{p}_{f}\right)}}{\left(\frac{1}{N_{|| \mathbf{p}_{i} \mid}^{2 \mathrm{pt}}} \sum_{\text {fixed }\left|\mathbf{p}_{i}\right|} C_{P P}^{2 \mathrm{pt}}\left(t, \mathbf{p}_{i}\right)\right)\left(\frac{1}{N_{\left|\mathbf{p}_{f}\right|}^{2 \mathrm{pt}}} \sum_{\text {fixed }\left|\mathbf{p}_{f}\right|} C_{P P}^{2 \mathrm{pt}}\left(t^{\prime}, \mathbf{p}_{f}\right)\right)},
$$

one can cancel the double cosh part. Here the averages are taken over the same absolute value of momenta, using the rotational invariance.

When either or both of $\mathbf{p}_{i}$ and $\mathbf{p}_{f}$ is zero, we can use

$$
\Delta_{t^{\prime}} C_{P V_{0} P}^{3 \mathrm{pt}}\left(t, t^{\prime} ; \mathbf{p}_{i}, \mathbf{0}\right)=C_{P V_{0} P}^{3 \mathrm{pt}}\left(t, t^{\prime} ; \mathbf{p}_{i}, \mathbf{0}\right)-C_{P V_{0} P}^{3 \mathrm{pt}}\left(t, t_{\mathrm{ref}} ; \mathbf{p}_{i}, \mathbf{0}\right),
$$

$\Delta_{t^{\prime}} \Delta_{t} C_{P V_{0} P}^{3 \mathrm{pt}}\left(t, t^{\prime} ; \mathbf{0}, \mathbf{0}\right)=C_{P V_{0} P}^{3 \mathrm{pt}}\left(t, t^{\prime} ; \mathbf{0}, \mathbf{0}\right)-C_{P V_{0} P}^{3 \mathrm{pt}}\left(t, t_{\mathrm{ref}} ; \mathbf{0}, \mathbf{0}\right)-C_{P V_{0} P}^{3 \mathrm{pt}}\left(t_{\mathrm{ref}}, t^{\prime} ; \mathbf{0}, \mathbf{0}\right)+C_{P V_{0} P}^{3 \mathrm{pt}}\left(t_{\mathrm{ref}}, t_{\mathrm{ref}} ; \mathbf{0}, \mathbf{0}\right)$,

and ratios

$$
\begin{gathered}
R_{V}^{2}\left(t, t^{\prime} ;\left|\mathbf{p}_{i}\right|, \mathbf{0}, q^{2}\right) \equiv \frac{\frac{1}{N_{\left|\mathbf{p}_{i}\right|}^{3 \mathrm{pt}} \sum_{\text {fixed }\left|\mathbf{p}_{i}\right|, q^{2}} \Delta_{t^{\prime}} C_{P V P}^{3 \mathrm{pt}}\left(t, t^{\prime} ; \mathbf{p}_{i}, \mathbf{0}\right)}}{\frac{1}{N_{\left|\mathbf{p}_{i}\right|}^{\mathrm{ppt}} \sum_{\text {fixed }\left|\mathbf{p}_{i}\right|} C_{P P}^{2 \mathrm{pt}}\left(t, \mathbf{p}_{i}\right)\left[-\Delta_{t^{\prime}} \partial_{t^{\prime}} C_{P P}^{2 \mathrm{pt}}\left(t^{\prime}, \mathbf{0}\right)+E\left(\mathbf{p}_{i}\right) \Delta_{t^{\prime}} C_{P P}^{2 \mathrm{pt}}\left(t^{\prime}, \mathbf{0}\right)\right]}} \\
R_{V}^{3}\left(t, t^{\prime} ; \mathbf{0}, \mathbf{0}, q^{2}=0\right) \equiv \frac{\Delta_{t} \Delta_{t^{\prime}} C_{P V P}^{3 \mathrm{pt}}\left(t, t^{\prime} ; \mathbf{0}, \mathbf{0}\right)}{-\Delta_{t} C_{P P}^{2 \mathrm{pt}}(t, \mathbf{0}) \Delta_{t^{\prime}} \partial_{t^{\prime}} C_{P P}^{2 \mathrm{pt}}\left(t^{\prime}, \mathbf{0}\right)-\Delta_{t} \partial_{t} C_{P P}^{2 \mathrm{pt}}(t, \mathbf{0}) \Delta_{t^{\prime}} C_{P P}^{2 \mathrm{pt}}\left(t^{\prime}, \mathbf{0}\right)}
\end{gathered}
$$

For $R_{V}^{2}$ in Eq. (4.W) we can interchange the role of initial and final states and include the case of $\left|\mathbf{p}_{i}\right|=0$ and $\left|\mathbf{p}_{f}\right| \neq 0$.

Taking further ratios, we obtain

$$
\begin{aligned}
& F_{V}^{1}\left(t, t^{\prime}, q^{2}\right) \equiv \frac{R_{V}^{1}\left(t, t^{\prime} ;\left|\mathbf{p}_{i}\right|,\left|\mathbf{p}_{f}\right|, q^{2}\right)}{R_{V}^{3}\left(t, t^{\prime} ; \mathbf{0}, \mathbf{0}, 0\right)} \\
& F_{V}^{2}\left(t, t^{\prime}, q^{2}\right) \equiv \frac{R_{V}^{2}\left(t, t^{\prime} ;\left|\mathbf{p}_{i}\right|, \mathbf{0}, q^{2}\right)}{R_{V}^{3}\left(t, t^{\prime} ; \mathbf{0}, \mathbf{0}, 0\right)}
\end{aligned}
$$

which should converge to the form factor $F_{V}\left(q^{2}\right)$ at large $t$ and $t^{\prime}$. The leading finite volume effect is thus canceled by these simple manipulations.

Note that the strategy of taking ratios is not new but has been widely used for various purposes, such as canceling the smearing effect, renormalization and so on. The remarkable difference from these conventional approaches is that the zero-mode effect is manifestly removed before taking the ratios. 
In this section, we have explicitly demonstrated the cancellation of the dominant finite volume effects from the zero-mode, in the two and three point correlators using the $\varepsilon$ expansion of ChPT. This means that even in the so-called $\varepsilon$ regime, where $M_{\pi} L \lesssim 1$, the vector form factor of the pion can be extracted without resorting to the complicated Bessel functions. Here we would like to emphasize that the use of the $\varepsilon$ expansion is just for separating the zero-mode from others. Even in the $p$ regime, the structure of the zero-mode contribution is unchanged, our ratio method should work.

\section{Results by JLQCD collaboration}

As mentioned in the previous section, our strategy is to keep a good chiral symmetry on the lattice, which suppresses the lattice artifacts, and use the combination of the observables insensitive to the pion zero-mode to have a good control of the finite volume effects.

To this end, we sample the gauge configurations of size $16^{3} \times 48$ generated with the Iwasaki gauge action and 2+1-flavors of the overlap quark action. The simulations are carried out at $\beta=2.3$, where the lattice cutoff $1 / a=1.759(8)(5) \mathrm{GeV}(a \sim 0.112(1) \mathrm{fm})$ is estimated by the data for the $\Omega$-baryon mass as an input. The lattice size in the physical unit is $L \sim 1.8 \mathrm{fm}$.

In this work, we reduce the up-down quark mass to $m a=0.002$, which roughly corresponds to $3 \mathrm{MeV}$ in the physical unit, and the pion mass at this value is $m_{\pi} \sim 99 \mathrm{MeV}$ [2Q], which is below the physical point. For the strange quark, we set its mass almost at the physical value, $m_{s} a=0.080$. In this set up the pions are in the $\varepsilon$ regime $\left(m_{\pi} L \sim 0.90\right)$, while the kaons are in the $p$ regime.

Along the Hybrid Monte Carlo updates, the global topological charge is fixed at the trivial topological sector $Q=0$. Since its effect is encoded in the pion zero-mode, the ratio of the correlators introduced in the previous section is independent of $Q$, at least, at the leading order of ChPT.

The correlation functions are calculated using the smeared sources with the form of exponential function. To obtain a good statistical signal, the so-called all-to-all propagator technique is employed, where the low-energy part of the correlator is calculated from 160 eigenmodes of the Dirac operator and averaged over different source points, while the higher-mode contribution is estimated stochastically with the dilution technique [B]]. For $\Delta_{t}$ for the zero-momentum correlator, we use the reference time-slice at $t_{\text {ref }} a=12(\sim 1 \mathrm{fm})$.

The auto-correlation time of the correlators is at most 7 trajectories. The statistical errors in the analysis are estimated by the jackknife method after binning data in every 20 trajectories.

Figure $\mathbb{W}$ presents our lattice data of $F_{V}^{1}\left(t, t^{\prime}, q^{2}\right)$ at $\left(\mathbf{p}_{i}^{2}, \mathbf{p}_{f}^{2}, \mathbf{q}^{2}\right)=(2,1,1)$ (top panel) and $F_{V}^{2}\left(t, t^{\prime}, q^{2}\right)$ at $\left(\mathbf{p}_{i}^{2}, \mathbf{p}_{f}^{2}, \mathbf{q}^{2}\right)=(1,0,1)$ (bottom panel). Here the momenta are labeled in the units of $2 \pi / L$. The ratio $F_{V}^{2}$ defined in Eq. (4.J4) is used when either initial or final spatial momentum is zero with $t_{\text {ref }} a=12$. To estimate $E(\mathbf{p})$, which is involved in the definition of $R_{V}^{1}$ in Eq. (4.8), we use the dispersion relation $E(\mathbf{p})=\sqrt{\mathbf{p}^{2}+m_{\pi}^{2}}$. We find a plateau for time separations where $t$ and $t^{\prime}$ are greater than 5 , which is also stable against the change of $t_{\text {ref }}$ in the range $9 \leq t_{\text {ref }} a \leq 12\left(t_{\text {ref }}\right.$ should satisfy $\left.t+t^{\prime}+t_{\text {ref }} \ll T\right)$. We fit the data to a constant; the fit results are shown in the plots as well as the fit range.

We plot $F_{V}\left(q^{2}\right)$ obtained at various $q^{2}$ in Figure 1 . They are obtained at various combinations of $\mathbf{p}_{i}$ and $\mathbf{p}_{f}$ listed in Table $\mathbb{W}$. For comparison, we also plot the data in the $p$ regime (at $m a=0.015$ ) 

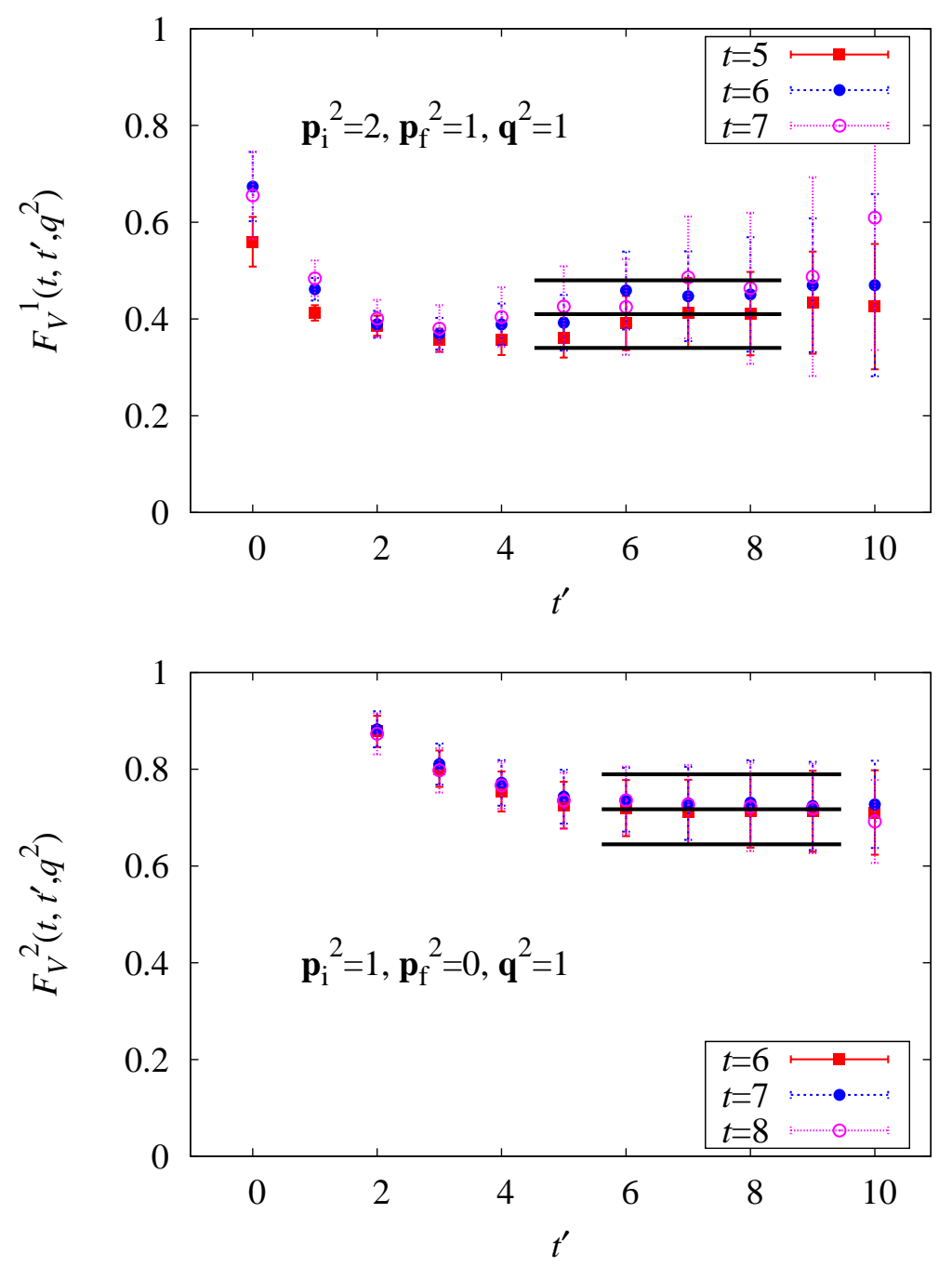

Figure 1: $F_{V}^{1}\left(t, t^{\prime}, q^{2}=-0.40\left[\mathrm{GeV}^{2}\right]\right)$ (top panel) and $F_{V}^{2}\left(t, t^{\prime}, q^{2}=-0.11\left[\mathrm{GeV}^{2}\right]\right)$ (bottom panel) are plotted as a function of $t^{\prime}$. The combination of initial and final momenta are shown in the plots in units of $2 \pi / L$. Data for different $t$ 's are plotted with different symbols. A constant fit and their error are shown by bands.

[B2]. The new data in the $\varepsilon$ regime show a steeper slope near the origin, which indicates a larger value of the pion charge radius.

We fit the form factor $F_{V}\left(q^{2}\right)$ to a function

$$
F_{V}\left(q^{2}\right)=\frac{1}{1-q^{2} / m_{V}^{2}}+a_{1} q^{2}+a_{2}\left(q^{2}\right)^{2}
$$

which is motivated by the vector dominance hypothesis and corrections are added as a polynomial. The same function was also used in our previous analysis of the $p$ regime data [ 3$]$. Since our calculation of the vector meson mass on the $\varepsilon$ regime ensemble is too noisy to be useful, we use the physical $\rho$ meson mass, $770 \mathrm{MeV}$, as an input to Eq. (5.J) and treat $a_{1}$ and $a_{2}$ as free parameters. The fit curve goes through the lowest four $\left|q^{2}\right|$ points as shown in Figure $\square$. The $\chi^{2} /$ dof is below 


\begin{tabular}{cccc}
\hline$(a q)^{2}$ & $\mathbf{p}_{i}$ & $\mathbf{p}_{f}$ & $\mathbf{q}$ \\
\hline 0.0380 & $(0,0,0)$ & $(1,0,0)$ & $(1,0,0)$ \\
0.0560 & $(0,0,0)$ & $(1,1,0)$ & $(1,1,0)$ \\
0.0699 & $(0,0,0)$ & $(1,1,1)$ & $(1,1,1)$ \\
0.1281 & $(0,1,0)$ & $(1,1,0)$ & $(1,0,0)$ \\
0.3084 & $(0,-1,0)$ & $(1,0,0)$ & $(1,1,0)$ \\
0.4366 & $(0,0,-1)$ & $(1,1,0)$ & $(1,1,1)$ \\
\hline
\end{tabular}

Table 1: Combinations of initial and final momenta taken in the calculation. The momentum components are given in units of $2 \pi / L$. Those equivalent under cubic rotations are averaged, though not listed.

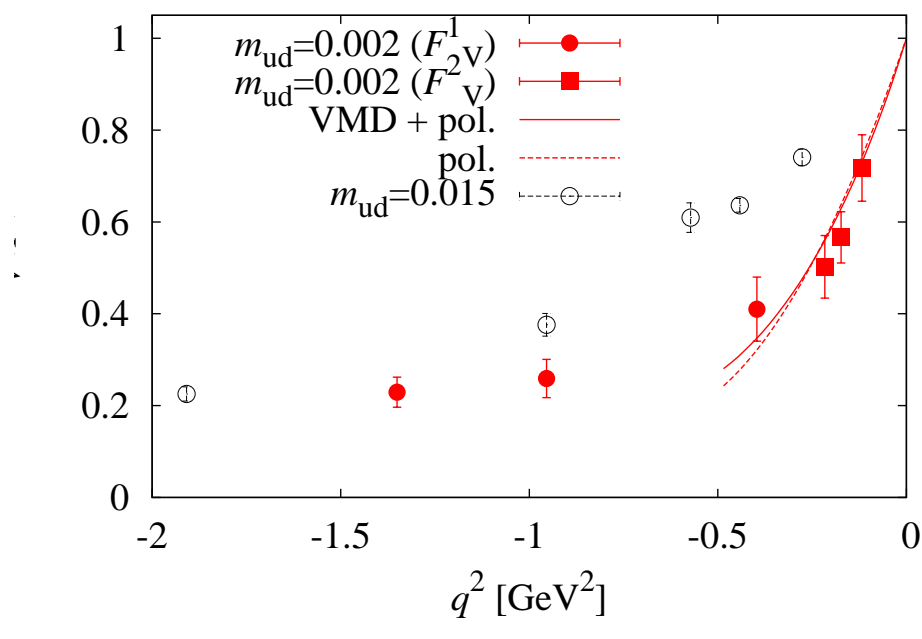

Figure 2: Lattice results for $F_{V}\left(q^{2}\right)$ as a function of $q^{2}$. The lattice data in the $\varepsilon$ regime ( $m_{u d} a=0.002$, filled symbols) and in the $p$ regime ( $m_{u d} a=0.015$, open symbols) are plotted. The $\varepsilon$ regime data are obtained either from the ratio $F_{V}^{1}$ (circles) or $F_{V}^{2}$ (squares).

1.0 in this case. When we include higher $\left|q^{2}\right|$ points, the fit becomes worse and $\chi^{2} /$ dof increases up to 2.5 .

The charge radius at our simulated mass is obtained as

$$
\left\langle r^{2}\right\rangle_{V}=0.63(08)(11) \mathrm{fm}^{2} \quad(\text { at } m=0.002),
$$

where the first error is statistical and the second is systematic, as explained below. Note that the central value is larger than the experimental value, $0.452(11) \mathrm{fm}^{2}$.

Although the main part of the pion zero-mode's effects is removed, the systematic error due to finite volume remains dominant. First, since the momentum space is discrete, the number of data points near $q^{2}=0$ is limited. The choice of the fitting range and/or fitting function affects the determination of the slope at $q^{2}=0$ by $12 \%$. Here we assign the variation of the fit results as the systematic effect. (The central value is taken from the fit of the lowest four $\left|q^{2}\right|$ point to Eq. (ل) (D).) In addition to the model function Eq. (5. لل), we attempt a simple polynomial function of second order (dashed curve in Figure 2 ) in this analysis. 


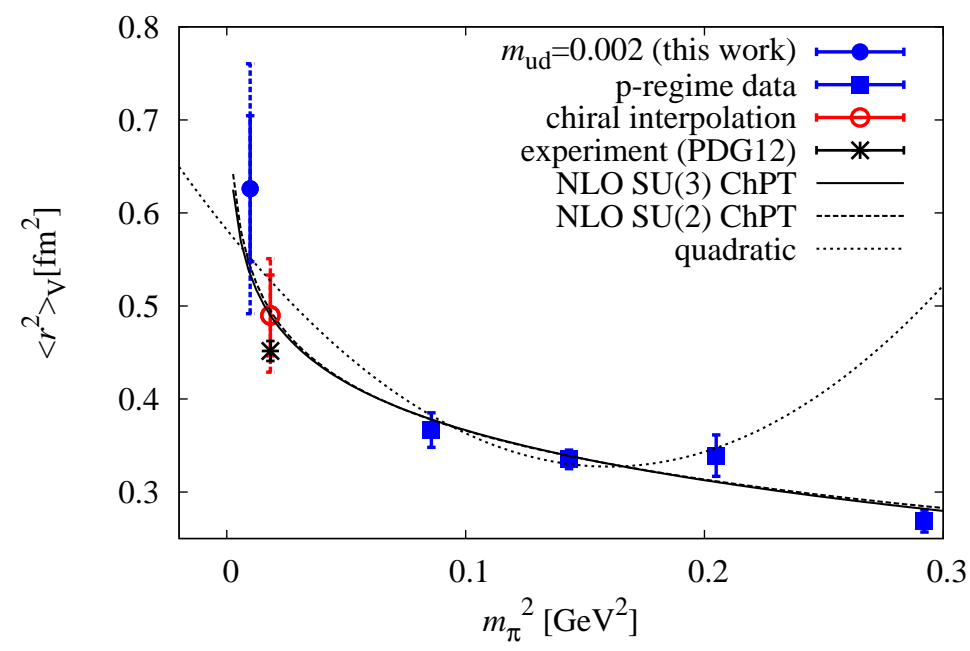

Figure 3: Pion charge radius as a function of $m_{\pi}^{2}$. A result from this work obtained in the $\varepsilon$ regime (circle) is plotted together with the data at heavier up and down quarks (square, from [32]). The experimental result is shown at $m_{\pi}=135 \mathrm{MeV}$. The fit curves are those of next-to-leading order ChPT as well as a simple polynomial (quadratic).

Second, the dispersion relation may be distorted in the $\varepsilon$ regime. By an estimate at the next-toleading order ChPT, it can be shown that a distortion of the form $E(\mathbf{p}) \rightarrow \sqrt{\mathbf{p}^{2}+Z_{m} m_{\pi}^{2}}$ with $Z_{m} \sim$ 2 is expected [ㅁ] ]. Since the relation $E(\mathbf{p}) \rightarrow \sqrt{\mathbf{p}^{2}+m_{\pi}^{2}}$ is used when constructing $R_{V}^{1}$, as given in Eq. (4.8), this effect may induce a bias as large as $\sim 10 \%$.

Finally, the effect of non-zero modes are non-negligible on our small lattice [2]]. We estimate its size as $8 \%$. In total, we assign $17 \%$ as the total size of the systematic error by adding these sources in quadrature. This is shown in Eq. (5.2) as our estimate of the systematic error. Because it is large, other sources, such as those from discretization effect, are expected to be sub-dominant.

Figure B] shows the dependence of $\left\langle r^{2}\right\rangle_{V}^{\pi}$ on the pion mass squared. The result Eq. (5.2) is plotted together with our previous calculation at heavier pions [B2]. It is clear that the $\varepsilon$ regime result (circle) is much higher than the points above $m_{\pi} \gtrsim 300 \mathrm{MeV}$ (square). It indicates the existence of the strong (logarithmic) curvature of the pion charge radius near the chiral limit.

Finally, we interpolate the data to the physical pion mass. We use the functions suggested by the $S U(2)$ and $S U(3) \mathrm{ChPT}$. At the next-to-leading order, they are

$$
\left\langle r^{2}\right\rangle_{V}^{\pi}=-\frac{1}{N F^{2}}\left(1+6 N \ell_{6}^{r}\right)-\frac{1}{N F^{2}} \ln \frac{m_{\pi}^{2}}{\mu^{2}}
$$

and

$$
\left\langle r^{2}\right\rangle_{V}^{\pi}=\frac{1}{2 N F^{2}}\left(-3+24 N L_{9}^{r}\right)-\frac{1}{N F^{2}} \ln \frac{m_{\pi}^{2}}{\mu^{2}}-\frac{1}{2 N F^{2}} \ln \frac{m_{K}^{2}}{\mu^{2}},
$$

for $S U(2)$ and for $S U(3)$, respectively, with $N=(4 \pi)^{2}$ and $F$ the pion decay constant in the chiral limit. The parameters $\ell_{6}^{r}$ and $L_{9}^{r}$ are relevant low-energy constants in $S U(2)$ and $S U(3)$ ChPT, 
respectively. The result for the charge radius at the physical pion mass is

$$
\left\langle r^{2}\right\rangle_{V}=0.49(4)(4) \mathrm{fm}^{2} \text { (at physical point), }
$$

where the first error is statistical and the second is systematic, including the one in Eq. (5.2) as well as the variation due to the choice of the chiral fit functions. That includes the ChPT formulas and a polynomial function at the second order.

Through the ChPT fits, we also obtain $F$ and $\ell_{6}^{r}$ (or $L_{9}^{r}$ ). The value for $F$ is lower than its physical value: 57(8)(10) MeV and 60(9)(9) MeV for the $S U(2)$ and $S U(3)$ fits. This is consistent with our previous extensive analysis of the pion mass and decay constant in two-flavor QCD [32]. The low-energy constants, in the conventional notations, we obtained in this analysis are

$$
\begin{aligned}
\bar{\ell}_{6}=-6 N \ell_{6}^{r}\left(\mu=m_{\pi}\right) & =7.5(1.3)(1.5), \\
L_{9}^{r}(\mu=770 \mathrm{MeV}) & =2.4(0.8)(1.0) \times 10^{-3} .
\end{aligned}
$$

These values are also smaller than their phenomenological estimates, which may indicate that NNLO corrections are not negligible, in our $p$ regime data points.

\section{NLO finite volume effects}

After removing the dominant finite volume effect from the zero-mode, what remains in $F_{V}\left(q_{0}, \mathbf{q}\right)$ is the effect of the non-zero momentum modes, which is expected to be perturbatively small. In this section, we compute this non-zero-momentum effect to the pion 1-loop and numerically confirm this expectation.

To this end, all we need to evaluate is

$$
I_{\mu v}\left(q_{0}, \mathbf{q}\right)=\frac{1}{V} \sum_{p \neq 0, q} \frac{-2 p_{\mu} p_{v}}{p^{2}(p-q)^{2}} .
$$

Here and in the following, we ignore the terms proportional to $q_{v}$, since they are always contracted with a perpendicular 4-momentum vector to $q_{\mu}$, and thus do not contribute to the final result.

It is not difficult to decompose it as

$$
I_{\mu v}\left(q_{0}, \mathbf{q}\right)=\sum_{b_{\mu}=n_{\mu} L_{\mu}} I_{\mu v}^{b}\left(q_{0}, \mathbf{q}\right)
$$

where

$$
I_{\mu \nu}^{b}\left(q_{0}, \mathbf{q}\right) \equiv \int \frac{d^{4} p}{(2 \pi)^{4}} e^{i p b} \frac{-2 p_{\mu} p_{v}}{p^{2}(p-q)^{2}}
$$

Note that $I^{b=0}\left(q_{0}, \mathbf{q}\right)$ is the infinite volume limit of $I_{\mu v}\left(q_{0}, \mathbf{q}\right)$ and thus, the finite volume correction is given by

$$
\Delta I_{\mu v}\left(q_{0}, \mathbf{q}\right)=\sum_{b \neq 0} I_{\mu v}^{b}\left(q_{0}, \mathbf{q}\right)
$$


In the standard manner, each contribution $I_{\mu \nu}^{b}\left(q_{0}, \mathbf{q}\right)$ can be computed as

$$
\begin{aligned}
I_{\mu v}^{b}\left(q_{0}, \mathbf{q}\right) & =2 \frac{\partial}{\partial b^{\mu}} \frac{\partial}{\partial b^{v}} \int_{0}^{1} d x e^{i x b q} \int \frac{d^{4} p}{(2 \pi)^{4}} \frac{e^{i p b}}{\left(p^{2}+\Delta\right)^{2}} \\
& =-\frac{1}{4 \pi^{2}} \int_{0}^{1} d x e^{i x b q}\left[\frac{\delta_{\mu v}}{\left|b_{\mu}\right|} \sqrt{\Delta} K_{1}\left(\sqrt{\Delta}\left|b_{\mu}\right|\right)-\frac{b_{\mu} b_{v}}{\left|b_{\mu}\right|^{2}} \Delta K_{2}\left(\sqrt{\Delta}\left|b_{\mu}\right|\right)\right]
\end{aligned}
$$

where $\Delta=x(1-x) q^{2}$, and $K_{i}(z)$ denotes the $i$-th modified Bessel function. Here, we have neglected a term proportional to $q_{\mu} b_{v}$, since that term is proportional to $q_{v}$ after the summation over $b_{v}$.

When $b_{0}=0$, it is straight forward to numerically evaluate the above form. However, when $b_{0} \neq 0$, we need a special care because we need to analytically continuate the results with respect to $q_{0}$. Here we simplify the situation using an inequality

$$
\left|\int_{0}^{1} d x e^{i \alpha} f(x)\right|<\left|\int_{0}^{1} d x\right| e^{i \alpha}|f(x)|=\left|\int_{0}^{1} d x f(x)\right|
$$

in Eq. (6.5). Namely we neglect the oscillating factor $\exp \left(i x b_{0} q_{0}\right)$. Then the analytic continuation of $q_{0}$ has no subtlety since the Bessel functions are all vanishing in the limit $\left|q_{0}\right| \rightarrow \infty$ with any complex phase. Note here that the real part $\sqrt{\Delta}$ is always positive. We do not think this overestimation affects the result very much, since the temporal direction is usually larger than the spatial direction by a factor of 2 or 3 , and therefore, the contribution from $b_{0} \neq 0$ is much smaller from the beginning.

Taking $\mu=0$ direction the finite volume correction to $F_{V}\left(q_{0}, \mathbf{q}\right)$ can be computed as

$$
\begin{aligned}
\Delta F_{V}\left(q_{0}, \mathbf{q}\right) & \equiv F_{V}\left(q_{0}, \mathbf{q}\right)-F_{V}^{\infty}\left(q_{0}, \mathbf{q}\right) \\
& =-\frac{N_{f}}{2 F_{\text {eff }}^{2}}\left(\Delta l\left(q_{0}, \mathbf{q}\right)-l_{00}\right),
\end{aligned}
$$

where

$$
\Delta l\left(q_{0}, \mathbf{q}\right)=-\frac{1}{4 \pi^{2}} \sum_{b_{\mu}} \int_{0}^{1} d x e^{i x \mathbf{b} \cdot \mathbf{q}}\left[\frac{\sqrt{\Delta}}{\left|b_{\mu}\right|} K_{1}\left(\sqrt{\Delta}\left|b_{\mu}\right|\right)-\frac{b_{0}^{2}}{\left|b_{\mu}\right|^{2}} \Delta K_{2}\left(\sqrt{\Delta}\left|b_{\mu}\right|\right)\right] .
$$

Note that $\Delta l(0, \mathbf{0})=l_{00}$.

Our numerical estimates for $\Delta F_{V}\left(q_{0}, \mathbf{q}\right)$ at $L=T / 2=2,3,4 \mathrm{fm}$ are presented in Fig. 田. Here, we denote $q^{0}=i\left(\sqrt{\mathbf{p}_{f}^{2}+M_{\pi}^{2}}-\sqrt{\mathbf{p}_{i}^{2}+M_{\pi}^{2}}\right)$, assuming the dispersion relation of the pion energy, $\mathbf{q}=\mathbf{p}_{f}-\mathbf{p}_{i}$, and choose $M_{\pi}=135 \mathrm{MeV}, F_{\text {eff }}=92.2 \mathrm{MeV}$, as inputs. The zig-zag behavior may be due to the lack of the rotational symmetry on the lattice. Since $F_{V}^{\infty}\left(q^{2}\right)$ is an $\mathscr{O}(1)$ quantity, our result shows the remaining finite volume effects is around a few $\%$ already at $L=3 \mathrm{fm}$, even when $m_{\pi} L<1$.

\section{Summary}

In this work, we propose a method to calculate the pion form factor in the $\varepsilon$ regime. Inserting momenta to the operators, and taking appropriate ratios of them, we can eliminate the dominant 


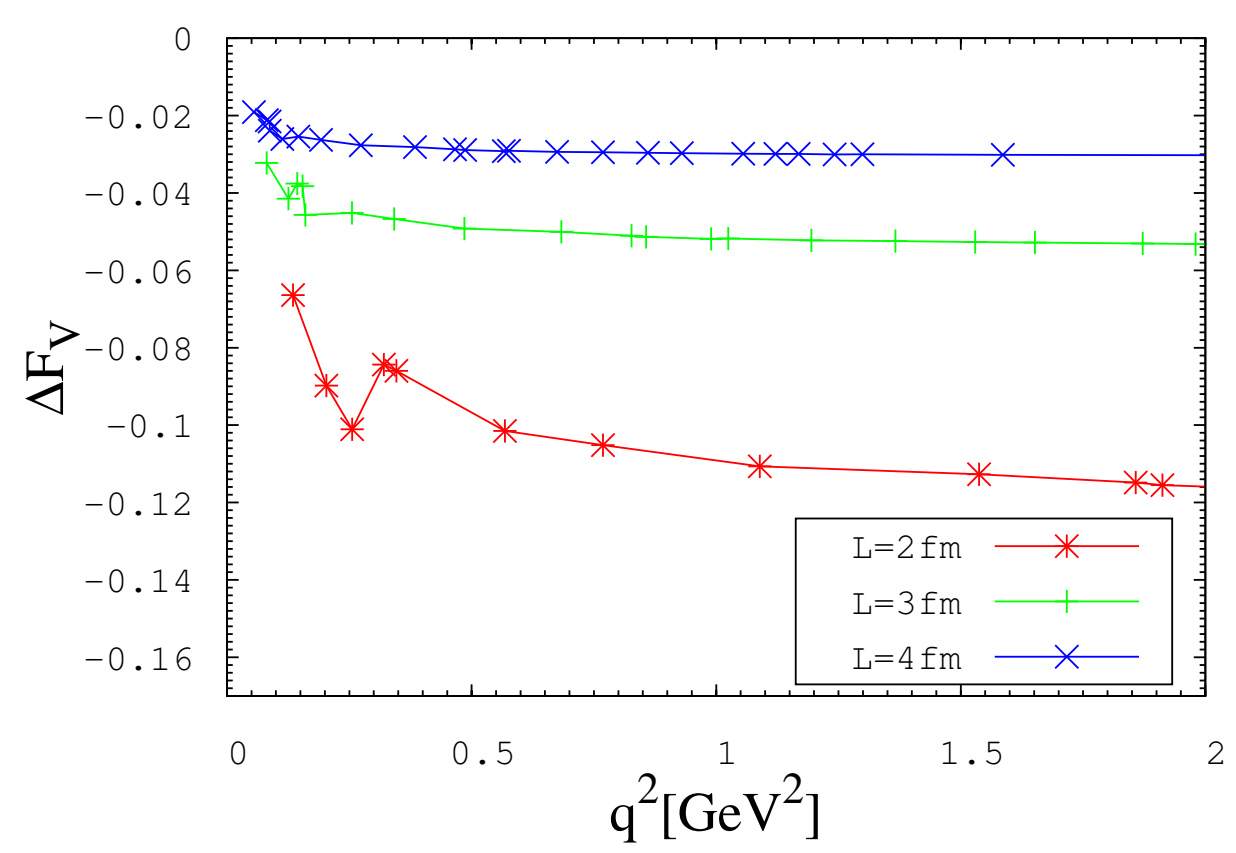

Figure 4: Numerical estimates for $\Delta F_{V}$.

contribution from the pion zero-mode. A tree-level analysis of the vector pion form factor in the $\varepsilon$ regime confirms this observation; the result for the pion charge radius is consistent with the experiment, showing the existence of a logarithmic divergence towards the chiral limit.

This cancellation of the zero-mode occurs only at the leading order, and there should be nontrivial corrections at the next-to-leading order. This remaining finite volume effects are turned out to be sizable in the presented calculation. On the lattice of size $L \sim 3 \mathrm{fm}$ or larger, such effect would be reduced to a few $\%$ level. One may also use the twisted boundary condition for the valence quarks, although we need a study of the partially quenched effect in the $\varepsilon$ regime analysis of ChPT.

We thank P. H. Damgaard and members of JLQCD collaboration for fruitful discussions. Numerical simulations are performed on the IBM System Blue Gene Solution at High Energy Accelerator Research Organization (KEK) under a support of its Large Scale Simulation Program (No. 10-11, 12-06, 12/13-04). This work is supported in part by the Grant-in-Aid of the Japanese Ministry of Education (No. 21674002, 25287046, 25400284, 25800147, 26247043), the Grant-in-Aid for Scientific Research on Innovative Areas (No. 2004:20105001), and MEXT SPIRE (Strategic Programs for Innovative Research) Field 5 and JICFuS (Joint Institute for Computational Fundamental Science).

\section{References}

[1] C. Hoelbling, Acta Phys. Polon. B 45, no. 12, 2143 (2014) [arXiv:1410.3403 [hep-lat]].

[2] J. Gasser and H. Leutwyler, Annals Phys. 158, 142 (1984). 
[3] J. Gasser and H. Leutwyler, Nucl. Phys. B 250, 465 (1985).

[4] B. B. Brandt, Int. J. Mod. Phys. E 22, 1330030 (2013) [arXiv:1310.6389 [hep-lat]].

[5] B. B. Brandt, A. Juttner and H. Wittig, PoS ConfinementX , 112 (2012) [arXiv:1301.3513 [hep-lat]].

[6] J. Koponen, F. Bursa, C. Davies, G. Donald and R. Dowdall, PoS LATTICE 2013, 282 (2014) [arXiv:1311.3513 [hep-lat]].

[7] H. Fukaya, S. Aoki, S. Hashimoto, T. Kaneko, H. Matsufuru and J. Noaki, Phys. Rev. D 90, no. 3, 034506 (2014) [arXiv:1405.4077 [hep-lat]].

[8] X. Feng, S. Aoki, S. Hashimoto and T. Kaneko, Phys. Rev. D 91, no. 5, 054504 (2015) [arXiv:1412.6319 [hep-lat]].

[9] B. Owen, W. Kamleh, D. Leinweber, B. Menadue and S. Mahbub, Phys. Rev. D 91, no. 7, 074503 (2015) [arXiv:1501.02561 [hep-lat]].

[10] J. Bijnens and J. Relefors, JHEP 1405, 015 (2014) [arXiv:1402.1385 [hep-lat]].

[11] K. A. Olive et al. [Particle Data Group Collaboration], Chin. Phys. C 38, 090001 (2014).

[12] C. Alexandrou, M. Constantinou, S. Dinter, V. Drach, K. Jansen, C. Kallidonis and G. Koutsou, Phys. Rev. D 88, no. 1, 014509 (2013) [arXiv:1303.5979 [hep-lat]].

[13] T. Bhattacharya, S. D. Cohen, R. Gupta, A. Joseph, H. W. Lin and B. Yoon, Phys. Rev. D 89, no. 9, 094502 (2014) [arXiv:1306.5435 [hep-lat]].

[14] M. Lin, PoS LATTICE 2013, 275 (2014) [arXiv:1401.1476 [hep-lat]].

[15] P. E. Shanahan et al. [CSSM and QCDSF/UKQCD Collaborations], Phys. Rev. D 89, 074511 (2014) [arXiv:1401.5862 [hep-lat]].

[16] P. E. Shanahan et al., Phys. Rev. D 90, 034502 (2014) [arXiv:1403.1965 [hep-lat]].

[17] J. R. Green, J. W. Negele, A. V. Pochinsky, S. N. Syritsyn, M. Engelhardt and S. Krieg, Phys. Rev. D 90, 074507 (2014) [arXiv:1404.4029 [hep-lat]].

[18] S. Capitani et al., Phys. Rev. D 92, no. 5, 054511 (2015) [arXiv:1504.04628 [hep-lat]].

[19] T. Bhattacharya, V. Cirigliano, R. Gupta, H. W. Lin and B. Yoon, arXiv:1506.04196 [hep-lat].

[20] T. Bhattacharya, V. Cirigliano, S. Cohen, R. Gupta, A. Joseph, H. W. Lin and B. Yoon, arXiv:1506.06411 [hep-lat].

[21] H. Fukaya and T. Suzuki, Phys. Rev. D 90, no. 11, 114508 (2014) [arXiv:1409.0327 [hep-lat]].

[22] J. Gasser and H. Leutwyler, Phys. Lett. B 184, 83 (1987).

[23] P. Hasenfratz and H. Leutwyler, Nucl. Phys. B 343, 241 (1990).

[24] S. Aoki et al., Eur. Phys. J. C 74, 2890 (2014) [arXiv:1310.8555 [hep-lat]].

[25] F. C. Hansen, Nucl. Phys. B 345, 685 (1990).

[26] P. H. Damgaard, M. C. Diamantini, P. Hernandez and K. Jansen, Nucl. Phys. B 629, 445 (2002) [hep-lat/0112016].

[27] F. Bernardoni, P. H. Damgaard, H. Fukaya and P. Hernandez, JHEP 0810, 008 (2008) [arXiv:0808.1986 [hep-lat]].

[28] S. Aoki and H. Fukaya, Phys. Rev. D 84, 014501 (2011) [arXiv:1105.1606 [hep-lat]]. 
[29] H. Fukaya et al. [JLQCD Collaboration], PoS LATTICE 2011, 101 (2011) [arXiv:1111.0417 [hep-lat]].

[30] J. Foley, K. Jimmy Juge, A. O’Cais, M. Peardon, S. M. Ryan and J. -I. Skullerud, Comput. Phys. Commun. 172, 145 (2005) [hep-lat/0505023].

[31] S. Aoki et al. [JLQCD and TWQCD Collaborations], Phys. Rev. D 80, 034508 (2009).

[32] T. Kaneko et al.[JLQCD Collaboration], PoS LATTICE2010, 146 (2010). 